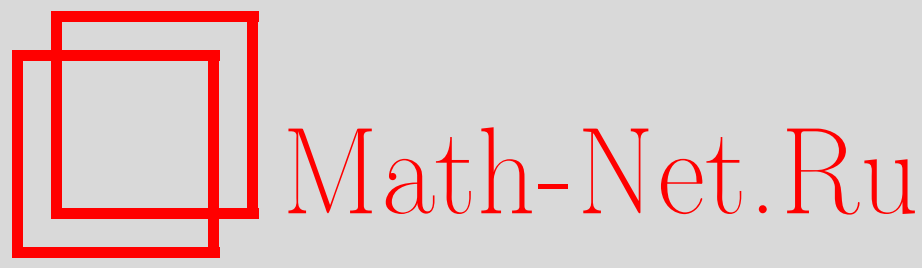

В. А. Кудрявцев, Тяжелые состояния адронной струны как слабо взаимодействующие тяжелые частицы темной материи, ТМФ, 2015, том 184, номер 3, 483-486

DOI: https://doi.org/10.4213/tmf8914

Использование Общероссийского математического портала Math-Net.Ru подразумевает, что вы прочитали и согласны с пользовательским соглашением http://www.mathnet.ru/rus/agreement

Параметры загрузки:

IP : 35.173 .137 .237

26 апреля 2023 г., $15: 27: 26$

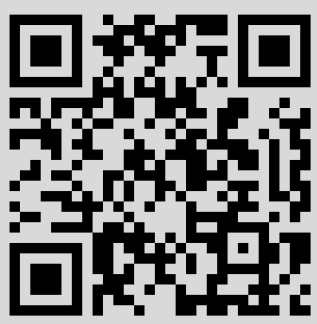




\title{
ФИЗИКА
}

Том 184, № 3

сентябрь, 2015

(C) 2015 г.

В. А. Кудрявцев*

\section{ТЯЖЕЛЫЕ СОСТОЯНИЯ АДРОННОЙ СТРУНЫ КАК СЛАБО ВЗАИМОДЕЙСТВУЮЩИЕ ТЯЖЕЛЫЕ ЧАСТИЦЫ ТЕМНОЙ МАТЕРИИ}

\begin{abstract}
Массивные состояния (с энергиями $\gtrsim 10$ ГэВ) адронной струны (с масштабом $\alpha^{\prime} \approx 1$ Гэ $\mathrm{B}^{-2}$ ) могут иметь очень малую константу связи с обычными барионами Вселенной. Время жизни для таких состояний оказывается порядка или даже больше возраста Вселенной. Предполагается, что эти тяжелые состояния могут быть кандидатами на роль слабо взаимодействующих тяжелых частиц темной материи.
\end{abstract}

Ключевые слова: адронная струна, темная материя, слабо взаимодействующие тяжелые частицы.

DOI: $10.4213 / \operatorname{tmf} 8914$

Струнные модели (называемые ранее дуальными резонансными моделями) [1], [2] рассматривались как подходящие модели для описания сильных взаимодействий на низких и промежуточных энергиях. В настоящий момент феноменологический статус такого подхода кажется даже более впечатляющим, чем сорок лет назад, поскольку сейчас струноподобный спектр адронных состояний включает не только ведущие реджевские траектории, но первые, вторые и даже третьи дочерние траектории для этого спектра [3], [4] вплоть до спинов $J=11 / 2$ для барионов. Однако ранее не были найдены формулы для последовательных (т. е. не содержащих в физическом спектре "духов", состояний с отрицательной нормой) струнных амплитуд взаимодействия произвольного числа адронов с пересечением ведущей мезонной реджевской траекторией, равной $1 / 2$ [5]. Теперь возможно построить модель составной струны, совместимой с этими требованиями [6]. Эта модель дает реалистическое описание адронного спектра и приводит к $\pi$-мезонным амплитудам взаимодействия с правильным энергетическим поведением, удовлетворяющим условию Адлера-Вайнберга [7].

Все модели релятивистских квантовых струн приводят к экспоненциальному росту числа струнных состояний по массе $m$ этих состояний. Ранее Хагедорн [8] получил такой рост для модели статистического бутстрапа и обнаружил соответствие

* Петербургский институт ядерной физики им. Б. П. Константинова НИЦ "Курчатовский институт", Санкт-Петербург, Россия. E-mail: via-kudryavtsev@yandex.ru 
такого поведения данным эксперимента для реальных адронных состояний. Согласно Хагедорну [9] эта зависимость числа адронных состояний на единицу массы $f(m)$ выражается асимптотически следующей формулой:

$$
f(m) \approx A m^{-\gamma} e^{m / m_{0}}
$$

В модели Хагедорна $\gamma=5 / 2, m_{0} \approx 0.16$ ГэВ. В струнном подходе эти параметры зависят от эффективной размерности $d$ для обычной классической струны:

$$
m_{0}=\frac{1}{\pi} \sqrt{\frac{3}{2 d \alpha^{\prime}}}
$$

В нашем случае модели адронной струны мы имеем $d=d_{\mathrm{bos}}+d_{\text {mes }} / 2=8+4=12$, $m_{0}=0.12$ ГэВ, $\alpha^{\prime} \approx(0.8-0.9)$ ГэВ ${ }^{-2}$.

Обсудим вклады этих тяжелых состояний в простые четырехточечные адронные амплитуды $A+B \rightarrow A+B: \pi+\pi \rightarrow \pi+\pi, \pi+N \rightarrow \pi+N, N+N \rightarrow N+N$ и т. д. Полюсной вклад в такую амплитуду для данной массы $m$ содержит сумму всех струнных состояний массы $m$. Мы можем видеть, что этот экспоненциальный рост числа состояний с ростом массы при степенном ограничении роста самой амплитуды приводит к экспоненциальному падению средней парциальной ширины $\bar{\Gamma}_{i}$ для этих состояний и соответствующих им констант связи с внешними адронами:

$$
\sum_{i} \Gamma_{i}=\Gamma, \quad \bar{\Gamma}_{i}=\frac{\Gamma}{f(m)}, \quad \bar{\Gamma}_{i} \approx \Gamma m_{\gamma} e^{-m / m_{0}} .
$$

Полная ширина Г растет с массой $m$ не быстрее, чем некоторая степень $m$. Таким образом, большая часть этих струнных состояний с большими значениями массы $m$ будет иметь исключительно малые ширины $\Gamma_{i}$ и, следовательно, весьма значительные времена жизни. Принимая $\Gamma \approx 1$ ГэВ, мы получим

$$
\bar{\tau}_{i} \approx \frac{1}{\bar{\Gamma}_{i}} \approx \frac{f(m)}{\Gamma} \approx 10^{-23} e^{m / m_{0}} \approx 10^{-23} e^{8.3 m} \mathrm{c} .
$$

Поскольку время жизни Вселенной $t_{0} \approx 13.7$ миллиардов лет, или $\approx 4.3 \cdot 10^{17} \mathrm{c}$, мы получим $\bar{\tau}_{i}>t_{0}$ для масс $m>11$ ГэВ.

Таким образом, эти тяжелые состояния с массами $m>11$ ГэВ могут рождаться в очень ранней Вселенной при $T>10^{14} \mathrm{~K}$ и спустя $t<10^{-8}$ с с момента Большого взрыва, они могут существовать в наше время. В силу изложенного выше они исключительно слабо взаимодействуют с обычными адронами и еще слабее друг с другом.

Оценки сечений взаимодействия этих состояний $A_{\mathrm{dm}}$ с обычными адронами $F A_{H}$ следуют из тех же соображений, что и выше:

$$
\sigma\left(A_{\mathrm{dm}}^{i}, A_{H}\right) \approx \frac{\sigma\left(A_{H}, A_{H}^{\prime}\right)}{f(m)} \approx \sigma_{H} e^{-8.3 m} .
$$

Эти оценки не противоречат экспериментальным данным по поискам частиц темной материи [10]. 
Обсудим теперь приведение такого рода тяжелых частиц в термодинамическое равновесие с окружающей средой. Исходя из изложенного выше можно заключить, что чрезвычайно мало взаимодействие рассматриваемых частиц с массой $m>10$ ГэВ как друг с другом, так и с другими более легкими адронами. Поэтому следует принять во внимание взаимодействие их с фотонами. Именно это взаимодействие существенно для приведения этих тяжелых частиц к термодинамическому равновесию с фотонным газом в эволюции Вселенной. В силу реакции $A_{\mathrm{dm}}+\bar{A}_{\mathrm{dm}} \leftrightarrow \gamma+\gamma$ фотоны будут обеспечивать термодинамическое равновесие с частицами темной материи до момента $t \sim 10^{-8}$ с. Далее, выйдя из равновесия с фотонным газом (предполагаем для этих частиц, как для продолжения обычных адронов, ту же барионную асимметрию $\sim 10^{-9}$ ), эти частицы, неспособные связаться в ядра, будут взаимодействовать исключительно гравитационно, образуя темную материю.

Из этих соображений для времен $t<10^{-8}$ с можно оценить вклад этих частиц в изменение со временем характерного масштаба Вселенной $a(t)$. При детальном равновесии этих частиц с фотонным газом $A_{\mathrm{dm}}+\bar{A}_{\mathrm{dm}} \leftrightarrow \gamma+\gamma$ при $t<10^{-8}$ с в кубе с характерным размером порядка комптоновской длины $R \sim 1 / m(h / 2 \pi=c=1)$ будет столько же фотонов с длиной волны $R$, сколько этих частиц с массой $m$.

Энергия тяжелых частиц с массой $m$ и фотонов составляет

$$
E \sim(\omega+m) f(m) \sim 2 m m^{-5 / 2} e^{m / m_{0}} A \sim 2 A m^{-3 / 2} e^{m / m_{0}}
$$

Это соответствует плотности энергии (очевидно, остальные вклады будут пренебрежимо малы)

$$
\epsilon \sim \frac{2 m^{-3 / 2} e^{m / m_{0}}}{R^{3}} \sim A m^{3 / 2} e^{m / m_{0}} .
$$

Отсюда можно найти уравнение, описывающее изменение масштаба Вселенной $a(t)$ в эпоху $t<10^{-8} \mathrm{c}, a \sim m^{-1}$, с помощью известного соотношения для критической Вселенной:

$$
\begin{gathered}
\epsilon=\frac{3(\dot{a} / a)^{2}}{8 \pi G}, \quad c=1, \\
\left(\frac{\dot{a}}{a}\right)=\sqrt{\frac{8 \pi G \epsilon}{3}}=\sqrt{\frac{8 \pi G}{3} 2 A a^{-3 / 2} e^{1 / a m_{0}}},
\end{gathered}
$$

или

$$
\dot{a}=4 \sqrt{\frac{\pi G}{3} A a^{1 / 4}} e^{1 / 2 a m_{0}} .
$$

Заметим, что для больших $t\left(t>1 / m_{0}\right)$ это уравнение дает $a \sim t^{4 / 3}$, а для малых $t$ ответ подобен известному инфляционному решению:

$$
a(t) \sim e^{\sqrt{\Lambda / 3 t}}
$$

Благодарности. Автор благодарен А. П. Бухвостову, Л. Н. Липатову и А. Н. Семенову за полезные обсуждения результатов работы. Исследование выполнено за счет гранта Российского научного фонда (проект № 14-22-00281). 


\section{Список литературы}

[1] J. Nambu, "Quark model and the factorization of the Veneziano amplitude", Symmetries and Quark Models (Wayne State University, Detroit, Michigan, June 18-20, 1969), ed. R. Chand, Gordon and Breach, New York, 1970, 269-278.

[2] M. B. Green, J. H. Schwarz, E. Witten, Superstring Theory, Cambridge Univ. Press, Cambridge, 1987.

[3] C. Lovelace, Phys. Lett. B, 28:4 (1968), 264-268; Д. В. Ширков, УФН, 102:9 (1970), 87-104.

[4] A. V. Anisovich, V. V. Anisovich, A. V. Sarantsev, Phys. Rev. D, 62:5 (2000), 051502, 5 pp.

[5] C. Lovelace, Nucl. Phys. B, 148:3-4 (1979), 253-282.

[6] В. А. Кудрявцев, Писъма в ЖЭТФ, 58:5, 321-325; V. A. Kudryavtsev, A. N. Semenova, Internat. J. Modern Phys. A, 27:29 (2012), 1250170, 23 pp.

[7] S. L. Adler, Phys. Rep., 137:4 (1965), B1022-B1033.

[8] R. Hagedorn, Nuovo Cimento A, 56:4 (1968), 1027-1057.

[9] J. H. Schwarz, Phys. Rep., 8:4 (1973), 269-335.

[10] R. Agnese, Z. Ahmed, A. J. Anderson et al. [CDMS Collab.], Silicon detector dark matter results from the final exposure of CDMS II, arXiv: 1304.4279. 